\title{
Analysis of reliability of power supply of consumers in non-full- phase modes using adaptive models of their calculation
}

\author{
T.N. Nasirov ${ }^{1}$, V.A. Nepomnyashchiy ${ }^{1}$, O.V. Radionova ${ }^{1}$ \\ ${ }^{1}$ Tashkent State Technical University named after Islam Karimov, 100095, Uzbekistan, Tashkent, University St. 2A
}

\begin{abstract}
Reviewed and analyzed issues related to the reliability of electricity supply in non-full-phase modes of electrical networks, to calculate which use adaptive methods, is built on one Central principle of modeling the modes of electric power systems (EPS). Special attention is paid to the calculation of damages from no full provided of electricity in non-full-phase modes.
\end{abstract}

\section{Introduction}

At damages (failures, incidents) of the main power supply equipment (power lines, groups of single-phase transformers, shunt reactors) in networks of $110 \mathrm{kV}$ and above which are working with effectively grounded neutral, the main part of shutdowns is connected with steady single-phase short circuits which share reaches on the average $65 \%$.

This creates an alternative:

1. For the period of repair of damaged equipment if you want to disconnect the damaged item at all, thus reducing network throughput with feasible energyeconomic consequences in the form of limits to some consumers, the undersupply of electricity to them and thereby causing economic damage or

2. Using single phase control switches or highlighting with disconnectors' help of damaged phase, and translate the work of a damaged element in incomplete phase mode "two wires - ground", leading to the emergence of a network components of currents and voltages of inverse and zero sequences, with the corresponding consequences in the form of possible overloads in the remaining phases and overheating of transformers, generators and motors currents reverse sequence. To prevent these overloads, it will be necessary limits the loads of consumers with the appearance no full provided of electricity and economic damage from power supply disruptions.

The duration of incomplete phase modes can range from a few hours (for repair of the overhead line phase or replacement of the transformer's phase) to several days and months (for factory repair of the damaged transformer's phase in the absence of the reserve phase) [1]. Operation in such modes is one, and often the only way to improve the reliability of the operation of the EPS and power supply of consumers in post-accident and repair modes.

For estimation the admissibility of non-full-phase modes of operation of the electric network with a grounded neutral, it is necessary: the first step - is to calculate the parameters of the normal mode and the parameters of the non-full-phase steady-state mode of the EPS, to determine the parameters of asymmetry in case of damage and subsequent shutdown of one of the phases;

the second step - is to identify the level of overcurrent of "healthy" phases, the degree of asymmetry of currents and the magnitude of the reverse sequence currents as the most dangerous for the occurrence of overheating of generators, transformers and motor load of consumers working in the network;

the third step - is the assessment of measures for unloading generating capacities and limiting the loads of consumers to remove re-loads from the network elements (generators, transformers, "healthy" phases of power lines).

To identify the economic efficiency of the use of non-full-phase modes of the EPS and to improve the reliability of the network, it is necessary:

the first - is to estimate the no full provided of electricity to consumers with a limited load and the resulting economic damage when operating in a nonfull-phase mode;

the second - is to determine the probability and frequency arising of non-full phase regimes;

the third - is to determine the likely damage to consumers when the electric network operates in a nonfull-phase mode: $\overline{\mathbf{3}}_{\text {Ущ }}^{\text {Нाтр }}$.

For comparison, you should do similar calculations for the alternative variant of the reliability of the network with disconnection of damaging element (transmission line, transformer) by, if it is necessary, measures to be introduced in allowable of mode settings area and entering the required limits of consumer loads.

Next, it is necessary to determine the under-supply of electricity and damage to consumers from violations of their power supply in the shutdown mode of the damaged network element, to assess the probability and 
frequency of these situations and the likely damage to

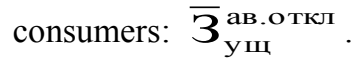

To select the option of doing network mode: unbalanced or disconnection of the damaged element, it is necessary to match the likely impact of the limitations of loads in unbalanced modes with the damages caused by outages of corrupted items:

$$
\overline{3}_{\text {ущ }}^{\text {нПтР }}</>\overline{3}_{\text {ущ. }}^{\text {ав.от }}
$$

and choose an option with minimal damage to consumers[2,3]:

$$
\min \left\{\overline{3}_{\text {ущ }}^{\text {НПР }}, \overline{3}_{\text {увш откл }}^{\text {ав.от }}\right\} .
$$

\section{Models of calculation of non-full-phase modes of EPS}

Mathematical models and algorithms for solving the problems of calculation and analysis of the modes of EPS, in addition to the general requirements of reliability, speed, convenience of representation of the source and output information, are subject to increasingly high requirements for the adequacy of the display of real modes, adaptability to changes in the network structure, modes and types of disturbances.

On the basis of the conducted analysis of existent methods of calculation of unbalance EPS [4-10] the conclusion about expediency of application for calculation and analysis of substitute complex schemes (SCS) based on symmetrical components.

Linear mathematical models of calculation of nonfull-phase modes of EPS are used for the purposes of relay protection and automation (RPA). Emergency modes of EPS caused both by longitudinal asymmetry, and modes of short circuits (SC) in the electric networks working in the incomplete phase mode are calculated.

The developed method of calculation of non-fullphase modes is based on the use of complex substitution schemes without direct electrical connections between the sequences.

For the formation of SCS, the substitution schemes of individual sequences of symmetric components in the places of asymmetries are connected to each other on the basis of boundary conditions. Modeling of the boundary conditions of asymmetry in the preparation of SCS is carried out with the help of multi poles communication (MPC) $[6,7]$ or sets of ideal transformers (IT) $[11,12]$ with transformation coefficients $K_{i t}=1, a, a^{2}$, where $a=$ $\exp (j 2 \pi / 3)$, corresponding to the coefficients of the equations of boundary conditions. These two different methods of modeling boundary conditions are based on fundamentally different approaches to the representation of asymmetry locations. In the first case the matrix of self and mutual conductancies of the place asymmetry; in the second case, with set of IT compiled boundary conditions of unbalance.

The use IT for communication between schemes of direct (DS), reverse (RS) and zero (ZS) sequences allows to provide the uniform principle of modeling of elements of a network within SCS. In addition, if the numbering of the SCS nodes is carried out so that the IT nodes receive the last numbers, then the matrix of the node conductivities of the SCS will have a block-diagonal structure with a bordering part in which the conductivities of the IT connections with the nodes of asymmetry are located. The block-diagonal structure of the SCS matrix allows avoiding the procedure of end-toend optimal renumbering of the complex circuit nodes in the analysis of non-full-phase modes and making maximum use of the results of calculations of the previous steady-state mode. At the same time, the advantages of calculating such matrices by methods of optimal elimination of variables are used to the maximum. The use of MPC for modeling of longitudinal type asymmetries violates the block-diagonal structure of the matrix of node conductivities of SCS due to the appearance of direct connections between the nodes of asymmetry of schemes DS, RS and ZS.

Based on the advantages of modeling the boundary conditions of IT over MPC, a universal algorithm for calculating incomplete-phase and complex-symmetric modes of EPS by complex substitution schemes is developed [12].

SCS, compiled for the calculation of non-full-phase and complex-symmetric modes of EPS, is described by a system of linear nodal equations:

$$
Y_{\mathrm{KC}_{3}} \dot{U}_{\mathrm{KC}_{3}}=\dot{J}_{\mathrm{KC}_{3}}
$$

where $\boldsymbol{Y}_{K C 3}$ - is the matrix of nodal conductivities of SCS;

$$
\dot{U}_{\mathrm{KC}_{3}} \text { - the desired vector of voltages of the }
$$
nodes of SCS;

$$
\dot{J}_{\mathrm{KC} 3} \text { - vector of nodal currents of SCS. }
$$

The structure of this system can be represented as follows:

$$
\left[\begin{array}{cccc}
Y^{(1)} & 0 & 0 & Y^{(1-D)} \\
0 & Y^{(2)} & 0 & Y^{(2-D)} \\
0 & 0 & Y^{(0)} & Y^{(0-D)} \\
Y^{(D-1)} & Y^{(D-2)} & Y^{(D-0)} & Y^{(D)}
\end{array}\right] *\left[\begin{array}{c}
\dot{U}^{(1)} \\
\dot{U}^{(2)} \\
\dot{U}^{(0)} \\
\dot{U}^{(D)}
\end{array}\right]=\left[\begin{array}{c}
\dot{J}^{(1)} \\
0 \\
0 \\
0
\end{array}\right],
$$

where $Y^{(1)}, Y^{(2)}, Y^{(0)}$ are the node conductance matrices of the DS, RS and ZS circuits, respectively;

$Y^{(i-D)}, Y^{(D-i)}$ - matrices of conductivity of connections MPC with nodes of asymmetry of PP, OP and NP ( $\mathrm{i}=$ $1,2,0) ; Y^{(D)}$ - matrix of nodal conductivities MPC;

$\dot{U}^{(1)}, \dot{U}^{(2)}, \dot{U}^{(0)}$ - vectors of node voltages of DS, RS and ZS circuits;

$\dot{J}^{(1)}$ - the vector of the driving currents in the nodes of the DS, determined by the values of the super-transient EMF generators obtained from the pre-calculated preemergency steady-state mode.

The system of linear equations of nodal stresses, describing SCS, with fixed values of super-transient EMF of generators and loads is solved by the method of ordered exclusion of unknowns (Gauss method). In addition, as a result of the solution, it is possible to 
directly determine the currents and voltages in the circuits of individual sequences.

The use of linear models of electrical systems used for the purposes of RPA for the calculation of non-fullphase steady-state regimes (NPSR) leads to large errors. NFRS are essentially load modes, so their calculation, as well as the calculation of the normal steady-state modes of power systems, should be carried out when setting the power values of load and generator units, that is, by nonlinear models.

Substitution schemes of individual sequences of DS, RS and ZS are formed on the basis of the same linear models, except for the account of loads and generators. As you know, in the coordinates of symmetric components synchronous generators are power sources only DS. Sources of currents and voltages of RS and ZS are places of asymmetries. Therefore, loads and generators in the calculation of NPSR are taken into account as follows: in the scheme of DS-the power of the normal (pre-emergency) mode, power losses in loads and generators caused by the flow of currents in them RS and ZS-the corresponding resistances.

The system of nonlinear nodal equations (4) is described. The equations of nodal stresses for DS nodes are nonlinear, since the driving currents in DS nodes are determined through the power of the nodes:

$$
\dot{J}_{i}^{(1)}=\hat{S}_{i} / \hat{U}_{i}
$$

where $\widehat{\mathcal{S}}_{i}$ and $\hat{\mathcal{U}}_{i}$ are the conjugate values of the power and voltage of the $i$-th node.

A method for solving a system of nodal equations describing the SCS is developed. Previously, the direct Gauss move excludes passive nodes of the RS and ZS circuits and additional it nodes. The result is a transformed system of nonlinear equations DS:

$$
Y_{*}^{(1)} \dot{U}^{(1)}=\left(\hat{U}_{(\text {diag })}^{(1)}\right)^{-1} \cdot \hat{S},
$$

where $\boldsymbol{Y}_{*}^{(1)}$ is the nodal conductance matrix of the extended (transformed) DS circuit;

$\left(\hat{U}_{(\text {diag })}^{(1)}\right)^{-1}$ - inverse diagonal matrix of conjugate stresses of DS nodes;

$\hat{S}$ - vector of conjugate powers of nodes.

Solution (6) is carried out by Newton-Raphson method for nodal equations in the form of current balance in a rectangular coordinate system. Further, the reverse Gauss stroke determines the voltage of the RS and ZS nodes.

The method of calculation of NPSR on complex schemes of substitution possesses sufficient simplicity of algorithm as is based on nodal equations, and universality for electric systems of any complexity. In addition, the obtained matrix $\boldsymbol{Y}_{*}^{(1)}$ is a matrix of nodal conductivities of the power system taking into account non-full-phase inclusions and can be used for the study of static aperiodic stability of NPSR.

\section{Probabilistic characteristics of non-full- phase modes and damage to consumers from violations of their power supply}

In unbalanced mode, de-energizing phase "A" and conservation in the "healthy" phases "b" and " $\mathrm{C}$ " determination of the coefficient of over-current in the remaining phases " $\mathrm{b}$ " and " $\mathrm{C}$ " is carried out according to the following formula:

$$
k_{\text {nep }}^{(\mathrm{I})}=\left\{\operatorname{abs}\left(I^{(B)}\right)-I_{B /}^{n p e d}\right\}_{>0} / I_{B J}^{n p e d},
$$

where $I_{B J}^{n p e d}$ is the maximum permissible heating current in the "healthy" phase.

The maximum asymmetry of the currents will be equal to the ratio of the total current in phase "B"(or in phase " $\mathrm{C}$ ") to the direct sequence current:

$$
\mathbf{k}_{\text {acus }}^{(\mathrm{I})}=I^{(\mathrm{B})} / I^{(1)},
$$

and the current asymmetry of the reverse sequence will be equal to:

$$
\mathrm{k}_{\text {acuM }}^{(2)}=I^{(2)} / I^{(1)} .
$$

These three factors will characterize the state of the network operating in the incomplete phase mode.

In [1] it is specified that at work of a network in the incomplete phase mode the asymmetry of currents in phases of generators shall not exceed the following values:

a) for turbogenerators $-12 \%$;

b) for hydrogenerators with air cooling of stator windings with a capacity of 125 MVA and below - 20\%, with a capacity of more than 125 MVA - 15\%, and for hydrogenerators with water cooling of stator windings $10 \%$;

c) for synchronous compensators - $20 \%$.

In this case, the reverse sequence current during long-term operation should not exceed 5\% for turbogenerators and $10 \%$ for hydrogenerators.

If switching measures to reduce the asymmetry of currents and currents of the reverse sequence do not give the desired results, it is necessary to limit the loads of consumers and reduce the overall load of overloaded power lines and generating equipment.

Unloading of the network should be carried out purposefully with the choice to limit the loads of those nodes that lead to current limitation in the most overloaded transmission line operating in incomplete phase mode. For this purpose it is necessary to use wellknown system of coefficients of distribution of currents $k_{(j)}^{(i)}$.

The current in the overloaded branch $\mathrm{j}$ is defined by the expression:

$$
I_{(j)}=\sum_{i=1}^{n_{y}} k_{(j)}^{(i)} \times I_{\mathrm{y}}^{(\mathrm{i})},
$$

where $I_{\mathrm{y}}^{(i)}$ is the resulting current in the $i$-th node.

To limit loads, nodes with coefficients that affect the unloading of the most overloaded j-th branch before the condition is met are selected: 


$$
I_{(j)}=\operatorname{abs}\left(\sum_{i=1}^{n_{y}} k_{(j)}^{(i)} \times I_{\mathrm{y}}^{(\mathrm{i})}\right)-I_{(\mathrm{j})}^{\text {пред }}=O,
$$

at the same time ensuring a minimum of damage to consumers according to the principles set out in $[3, \S$ 2.3].

The degree of load limitation of nodes to eliminate the overload of power lines operating in non-full-phase mode is defined as:

$$
\varepsilon_{i}^{o \text { гр }}=\mathrm{k}_{\text {nep }}^{(\mathrm{I})}=\left\{a b s\left(I^{(B)}\right)-I_{B I}^{n p e d}\right\}_{>0} / I_{B J}^{n p e d} .
$$

If the current asymmetry coefficients modulo (8) and the reverse sequence (9) are greater than their limit (normative) values, it will require additional limitation of consumer loads in the network nodes until the condition is reached:

$$
\mathrm{k}_{\text {acus }}^{(\mathrm{I})} \leq \mathrm{k}_{\text {nped }}^{(\mathrm{I})} ; \quad \mathrm{k}_{\text {acuм }}^{(2)} \leq \mathrm{k}_{\text {nped }}^{(2)} .
$$

With this in mind, the limited load nodes will be:

$$
\Delta P_{\text {нагр }}^{\max }=\varepsilon_{i}^{\text {orp }} \mathbf{P}_{\text {нагр }_{\mathrm{i}}}^{\max },
$$

and the system load limit will be equal to the sum of the node constraints:

$$
\Delta P_{\text {cuсm }}^{\text {oгp }}=\sum_{\mathrm{i}} \Delta P_{\text {нагр }}^{\max } .
$$

Slightly more complicated is the determination of the limits of the consumers in case of unbalance in open distribution equipment (ODE) nodes due to system failures or tire Assembly of switches, which leads to a transition in unbalanced mode all connected to this switchyard, power transmission lines and transformers. The difficulty lies in the fact that the restrictions of the loads of substations are fed from these power lines have an impact not just one but several working in unbalanced mode transmission lines. To do this, it is necessary to rank all substations connected with the damaged node according to the values of their influence on overload and current asymmetry in this node (ODE) and sequentially introduce load restrictions until the condition (13) is met.

Probabilistic characteristics of non-full-phase modes are:

1. The average annual frequency of single-phase steadystate power lines on the power lines entering the circuit and communication transformers (the latter, in principle, can be neglected, given their extremely rare damage relative to the power lines):

$$
\lambda_{\mathrm{ycT}}^{\mathrm{aв}(1)}=\mathrm{k}_{\mathrm{\kappa .3.}}^{(1)} \lambda_{\mathrm{yc \textrm {cT }}}^{\mathrm{aв}}, 1 / \text { year, }
$$

where $\mathrm{k}_{\mathrm{K} .3 \text {. }}^{(1)}$ - the share of single-phase SC in the total damage of the element (in the calculations, you can take the values of this coefficient for power lines- 0.65 and for OPC-0.60).

2. Mean time to restore (repair) of single-phase failure of an element, $\tau_{\text {к.3. }}^{\text {ав }(1)} \approx 3-6$ hours/off;

3. The probability of all emergency power lines, yelling at single-phase short-circuit:

$$
\mathrm{q}_{\kappa .3 .}^{(1)}=\lambda_{y c m}^{a \beta(1)} \tau_{\kappa .3 .}^{a \beta(1)} / 8760 \text {, Rel.units }
$$

Undersupply of electricity to consumers will be determined by the expression:

$$
\Delta \Im_{\kappa .3 .}^{a \beta(1)}=8760 \sum_{\mathrm{j}=1}^{\mathrm{N}} \varepsilon_{i}^{o z p} \Delta P_{\text {mazp }}^{\max } q_{\kappa .3 .3}^{(1)} \gamma_{\mathrm{i}}\left(\varepsilon_{i}^{o \rho p}\right),
$$

million $\mathrm{kWh}$,

where $\gamma_{\mathrm{i}}\left(\varepsilon_{i}^{o z p}\right)$ is the density of the bounded part of the load graph of the $i$-th node as a function of the degree of restriction of its load.

At the same time, the damage to consumers from violations of their power supply to ensure the functioning of the network with permissible asymmetry parameters will be:

$$
3_{y u, . \kappa .3 .}^{a \varepsilon(1)}=8760 \sum_{\mathrm{j}=1}^{\mathrm{N}} \varepsilon_{i}^{o z p} \Delta P_{\text {нагр }}^{\max } q_{\kappa .3 .}^{(1)} \gamma_{\mathrm{i}}\left(\varepsilon_{i}^{o z p}\right) 3_{y u_{\mathrm{i}}}^{a \beta}\left(\varepsilon_{\mathrm{i}}^{o z p}\right),
$$

million rubles / year,

Where $3_{y u_{\mathrm{i}}}^{a s}\left(\varepsilon_{\mathrm{i}}^{o z p}\right)$ - specific damage from violations of power supply of consumers of the $i$-th node, rub/ $\mathrm{kWh}$, taken according to [3].

As shown by preliminary calculations for several circuits of electrical networks economically expedient to use in stable single-phase SC incomplete phase mode than to work with the disconnection of damaged elements.

\section{Conclusion}

1. The use of adaptive nonlinear models for calculation of non-full-phase steady-state modes of EPS allows to significantly increase the accuracy of calculations.

2. Proposed model of electric networks of $110 \mathrm{kV}$ and above, taking into account the reliability of power supply allows to estimate the parameters of unbalance when single-phase short circuits in networks with grounded neutral and the resulting asymmetry of the currents in the healthy phases on the module and the currents reverse order and carry out the input modes allowable under asymmetry region by selectively targeted restrictions loads of customers in compliance with terms of at least economic damages.

3. Comparison of results of work of electric networks of $110 \mathrm{kV}$ and above in incomplete phase modes and their work with shutdown of the damaged elements allows to justify economically the most effective mode of their functioning in the emergency situations arising at steady single-phase short circuits.

\section{References}

1. Methodical instructions on application of incompletephase modes of operation of the main electrical equipment of electrical installations of 330-1150 kV. M.: ORGRES, 1999.

2. Nepomnyashchiy V.A. Economic and mathematical model of reliability of power systems and their electric networks //Electricity, 2012, №2, pp.5-16. 
3. Nepomnyashchiy V.A. Economic losses from power supply violations. -Moscow: Publishing house MEI, 2010, 190 p.

4. Losev S.B., Chernin A.B. Calculations of electrical quantities in asymmetric modes of electrical systems. Moscow: EAI, 1983.

5. Dunaeva N.P., Kontorovich A.M., Shcherbachev O.V. Method of calculation of non-full-phase steady-state modes of complex electrical systems: Proceedings of LPI, 1980, No. 369, pp. 3-5.

6. Sokolov N.I. Construction and application of complex substitution schemes for complex asymmetric circuits //Electricity, 1949, №8, pp. 21-28.

7. Popov V.A. Research and development of methods of calculation of electric quantities for relay protection at complex damages in electrical systems: diss. cand. tech. sciences. -Moscow: MEI, 1972, 32 p.

8. Berman A.P. Development and implementation of the method of analysis of asymmetric modes of complex electrical systems using phase coordinates: diss. cand. tech. sciences. -Moscow: VNIIE, 1984, 18 p.
9. Ragozin A.A., Misrikhanov M.Sh., Popov V.A., Yakimchuk N.N., Medov R.V. Modeling of electric power systems in solving problems of asymmetric modes // Improving the efficiency of power systems: Proceedings of IHEU, Vol. 4, -Moscow: Energoatomizdat, 2001, pp. 51-62.

10. Misrikhanov M.Sh., Ragozin A.A., Popov V.A., Kushkova E.I. Method of calculation of steady-state incomplete-phase modes of equipment $110 \mathrm{kV}$ and above in electrical systems // Improving the efficiency of power systems: Proceedings of IHEU, Vol. 4, -Moscow: Energoatomizdat, 2001, pp. 62-69.

11. Fazylov H.F., Nasyrov T.H., Tyncherova E.L., Radionova O.V. Modeling of complex symmetric regimes in problems of emergency control and reliability of power systems // Mathematical modeling in power engineering: Abstracts of the all-Union scientific and technical conference, Part 4, Kiev, 1990, p. 142.

12. Nasirov T.H., Radionova O.V. Modeling of modes of electrical systems. -T.: Fan va texnologiya, 2016, 336 p. 